\title{
Correction to: Transcutaneous Electrical Acupoint Stimulation Combined with Dexamethasone and Tropisetron Prevents Postoperative Nausea and Vomiting in Female Patients Undergoing Laparoscopic Sleeve Gastrectomy: a Prospective, Randomized Controlled Trial
}

\author{
Qiuju Xiong ${ }^{1} \cdot \mathrm{Su} \mathrm{Min}^{1}$ D $\cdot \mathrm{Ke} \mathrm{Wei}^{1} \cdot$ Yanmei Yang ${ }^{2} \cdot$ Jingyue Ma $^{1} \cdot$ Dan Liu $^{1} \cdot$ Menghua Zeng $^{3} \cdot$ Lei Zou $^{1}$
}

Published online: 3 August 2021

๑) Springer Science+Business Media, LLC, part of Springer Nature 2021

Correction to: Obesity Surgery (2021) 31:1912-1920

https://doi.org/10.1007/s11695-020-05205-9

In the original article the funding information was incomplete. The complete funding statement follows:

This work was supported by the Collaborative Fund of Chongqing Municipal Health and Health Committee and the Chongqing Science and Technology Bureau of China (grant 2019ZY3407), and supported by the Chongqing Health Appropriate Technology Promotion Project (grant 2021jstg026) and the Central Special Fund for Guiding Local Science and Technology Development (grant No.015).

Publisher's Note Springer Nature remains neutral with regard to jurisdictional claims in published maps and institutional affiliations.
The original article can be found online at https://doi.org/10.1007/ s11695-020-05205-9.
Lei Zou

zoulei4038@163.com

1 Department of Anesthesiology, The First Affiliated Hospital of Chongqing Medical University, Youyi Road 1, Yuzhong District, Chongqing 400016, People's Republic of China

2 College of Traditional Chinese Medicine, Chongqing Medical University, Chongqing 401331, People's Republic of China

3 Department of Gastrointestinal Surgery, The First Affiliated Hospital of Chongqing Medical University, Chongqing 400016, People's Republic of China 